\title{
Patrimonio fílmico informativo: hacia una red temática de acceso abierto y proyección iberoamericana (Cinedocnet)
}

\author{
Film heritage information: an open access Iberoamerican thematic network (Cinedocnet)
}

\author{
Alfonso LóPEZ YePES \\ Servicio Documentación Multimedia. Facultad Ciencias de la Información, \\ Universidad Complutense de Madrid, España, alopez@ucm.es
}

\begin{abstract}
Resumen
Proyecto de establecimiento de una red temática sobre patrimonio fílmico iberoamericano en estrecha relación con el ámbito informativo, en particular con los medios de comunicación. Se trata de una red informativa y de conocimiento cinematográfico, que pretende destacar la aportación cultural, sociológica, antropológica, literaria, filosófica, semiótica... del cine, de la información y documentación cinematográficas, de la investigación y gestión digital aplicada al ámbito fílmico, y de su difusión informativa, partiendo de las fuentes de información cinematográfica españolas en una primera fase, ampliable en una fase posterior al espectro iberoamericano. En dicha primera fase se ha diseñado un portal que contempla y persigue la construcción social del conocimiento cinematográfico basado en la interacción entre distintos grupos multidisciplinares de profesionales relacionados y su vinculación con diferentes comunidades de usuarios interesados en el tema cinematográfico. La investigación se encuentra fundamentada en una plataforma informática de acceso abierto, creada a partir de un programa-sistema de indexación de contenidos pluridisciplinares cinematográficos, al mismo tiempo polivalente para la recuperación de diversas tipologías de fuentes de consulta y referencia en diversos formatos y soportes.
\end{abstract}

Palabras clave: Documentación cinematográfica. Fuentes de información cinematográfica. Cinedocnet. Difusión cinematográfica. Patrimonio fílmico. Conocimiento fílmico. Iberoamérica. España.

\section{Introducción}

Ante la hipótesis de si es posible establecer una red informativa de patrimonio fílmico, de ámbito iberoamericano, en línea y con filosofía de red social... el estado de la cuestión que hemos estudiado nos sugiere plantearnos la siguiente pregunta: ¿existe patrimonio fílmico en red?. Dicho planteamiento de base nos remite a la consulta de las fuentes especializadas existentes y sus antecedentes; a la situación que presenta el acceso abierto en estos momentos de pleno desarrollo de la sociedad-red; o a la existencia de propuestas muy recientes y muy rela-

\begin{abstract}
Project for the establishment of a thematic network on the Ibero-American film heritage from an informative and media-oriented perspective, highlighting the cultural, sociological, anthropological, literary, philosophical and semiotic contribution of cinema, and film digital information management and dissemination. They project will start with Spanish sources at an early stage, and will be expandable to the whole Ibero-American area. In this first phase, a portal has been designed that enhances the social construction of cinematographic knowledge throughout the interaction among the various multidisciplinary groups of professionals and the different communities of users interested in film knowledge. The portal is based on a open access platform, and allows for the versatile and multidisciplinary retrieval of the wide scope of film sources, formats and media.
\end{abstract}

Keywords: Film research. Film information sources. Cinedocnet. Film information. Film heritage. Film knowledge. Latin America. Spain.

cionadas con el conocimiento cinematográfico, como la propuesta de una red de patrimonio informativo iberoamericano de archivos sonoros y audiovisuales (Redauvi), en función de la demanda de sectores profesionales, de formación e investigación, de la industria cinematográfica y de servicios (López, 2008).

Cinedocnet se alimenta de documentos fílmicos procedentes -y que remiten - a ámbitos informativos y de investigación emparentados con el cine; y que guardan una relación muy estrecha con la prensa, radio, televisión, publicidad, internet, redes sociales e incluso con las bibliotecas y 
servicios audiovisuales universitarios. Una muestra de las tipologías mencionadas se despliegan a lo largo de estas páginas. Conlleva la producción, recuperación y difusión de recursos informativos audiovisuales (cinematográficos más concretamente), extendida asimismo a ámbitos de comunicación 2.0 como son en la actualidad, entre otros, las ya aludidas bibliotecas y servicios universitarios, en función de la sociedad-red en que nos encontramos inmersos. La propuesta de red se apoya en una primera fase en un portal que anuncia la construcción social del conocimiento basado en la interacción entre distintos grupos multidisciplinares de profesionales con diferentes comunidades de usuarios, lo que desembocará, en una fase subsiguiente, en la red de patrimonio fílmico informativo.

El portal está soportado en una plataforma informática de acceso abierto, creada a partir de un programa o sistema de indexación de contenidos multidisciplinares y polivalente para la recuperación de fuentes de información cinematográfica, capaz al mismo tiempo de establecer un sistema de metadatos para la descripción de dichos contenidos fílmicos. A las posibilidades de recuperación del sistema se añade otra posibilidad, la de informar a la comunidad de usuarios reales y potenciales a través de sus canales de sindicación. $Y$, en fin, la de establecer un plan de divulgación científica con la utilización del sistema de correo masivo mediante la herramienta webcrawler Mbot, lo que supondrá una importante repercusión en la afiliación de cuentas de las redes sociales, tanto generalistas como específicas, sobre comunicación audiovisual y cinematográfica. La existencia de una gran cantidad de fuentes de información y su recuperación efectiva precisa de una metodología de descripción normalizada, que se adecue a los contenidos disponibles en Cinedocnet. Con este objeto se ha diseñado un sistema de metadatos que permite la recuperación efectiva de los mismos y que responde al nombre de Indexator (López, 2014a).

\section{Conceptualización, origen y proceso evolutivo de Cinedocnet}

Habitualmente, cuando se habla de patrimonio cinematográfico, se está refiriendo fundamentalmente al ámbito de la conservación, preservación y recuperación del cine; es decir, especialmente del ámbito representado por las filmotecas, cinetecas y cinematecas y las labores que en ellas se llevan a cabo, centradas fundamentalmente en el estudio e investigación sobre procesos químicos y biológicos de los materiales que confluyen en su degradación (Amo, 2006, 2012), y sobre la conservación, restaura- ción y difusión del patrimonio cinematográfico español (Camarero, 2012).

La película sigue siendo hoy el soporte cinematográfico por excelencia. Sin embargo, como todo lo físico, se deteriora y descompone, lo que conlleva la desaparición de unas obras que han registrado y representado la memoria de más de un siglo de vida del cine. En este sentido "Rescatando Sombras" documenta la labor de las filmotecas por rescatar de la muerte y el olvido estas obras, reconstruyéndolas, restaurándolas y conservándolas. (Franco, 2013).

Y otros autores aluden (Oliván, 2000) al depósito de las películas cinematográficas en España tanto en materia de legislación como en su aplicación práctica, constatándose que, pese a las variaciones sufridas a lo largo del tiempo en el ámbito legal, nunca se ha efectuado el depósito de películas: Para la salvaguarda y conservación del patrimonio cinematográfico, resulta necesaria la inclusión del depósito de películas cinematográficas en la legislación sobre el depósito legal.

Por otra parte, el proyecto contempla un concepto de patrimonio cinematográfico estrechamente relacionado con el ámbito informativo, de medios de comunicación $\mathrm{y}$, todavía más ampliamente, con el ámbito cultural. En consecuencia, qué se entiende por patrimonio, cómo se concibe el término en el ámbito especializado, a qué responde, en fin, el concepto de "patrimonio fílmico informativo", en suma, su conceptualización. Patrimonio audiovisual, y por ende cinematográfico, puede entenderse como localizaciónrecuperación-conservación-preservación; como documentación o información del y sobre el ámbito cinematográfico como apoyo informativo; como investigación o creación de bases de datos sobre aspectos relacionados, proyectos de investigación, formación; como difusión o exhibición de obras audiovisuales, divulgación y uso de los materiales de archivo en filmotecas, cinetecas y archivos audiovisuales de medios de comunicación y empresas informativas; como divulgación de las funciones y actividades de dichos medios. Concepto "informativo" como sinónimo de presencia y connivencia en el ámbito de los medios de comunicación (Monasterio, 2005).

Otros autores abundan asimismo en esta misma idea de patrimonio fílmico, de función de las filmotecas en cuanto a su labor expresamente cultural, independientemente de las tareas de conservación, recuperación y restauración cinematográfica. El motivo, claro y convincente, es la función a la que se enfrentan hoy las filmotecas. El intenso cambio digital que está atrave- 
sando el mundo del cine plantea importantes retos de futuro a las filmotecas, así como las consiguientes líneas de expansión que se dibujan en el actual escenario digital para el ámbito filmotecario (García, Alberich, 2014).

Por otra parte, ante la hipótesis de si es posible establecer una red de patrimonio fílmico informativo, de ámbito iberoamericano, en línea y con filosofía de red social, surge como respuesta a ello, como primera fase de trabajo, el diseño en 2012 de un blog-portal con el nombre de Cinedocnet $(2012,2014)$. Dicho sitio, al que se le dota inmediatamente de dominio propio se administra, mantiene y actualiza en el ámbito del Servicio de Documentación Multimedia (2014) del Departamento de Biblioteconomía y Documentación de la Universidad Complutense de Madrid. Pretende, en suma, responder a la siguiente pregunta: ¿existe patrimonio fílmico en red?. Cuestionamiento que desde el Servicio mencionado se apuesta por hacer en función de la experiencia investigadora y desarrollos realizados durante sus ya largos años de existencia y actuaciones (Servicio Documentación Multimedia, 1993-2014; Torrado, 2007), y todavía más concretamente en trabajos elaborados por el autor con anterioridad, precisamente en este mismo ámbito de publicación (López, 2008).

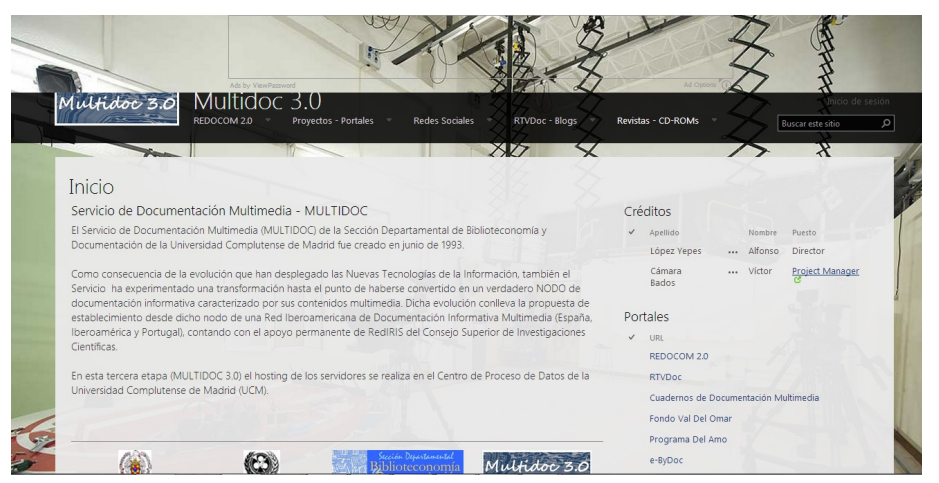

Figura 1. Servicio de Documentación Multimedia

Planteamiento que nos remite al estudio del estado de la cuestión en relación con el tema: a la consulta de las fuentes especializadas existentes y sus antecedentes (López, 2013a, 2013b); a la situación que presenta el acceso abierto en plena sociedad-red; o a la existencia, en fin, de propuestas muy recientes y estrechamente relacionadas, como la propuesta de una red de patrimonio iberoamericano de archivos sonoros y audiovisuales (Redauvi) (s. a.) con la que Cinedocnet mantiene, por sus contenidos, una relación muy estrecha en el año 2013 fecha de propuesta de esta otra red (Brausin, López, Rodríguez, 2012; López, Arratia, Brausin, De Giusti, Martínez, Rodríguez, Suárez, 2014; Ro- dríguez, Arratia, López, Brausin, De Giusti, Martínez, Suárez, 2013).

Asimismo, las fuentes de información consultadas previamente a propósito del establecimiento del portal (Cinedocnet), describen un estado de la cuestión favorable a su puesta en funcionamiento, que aglutine, integre, produzca, recupere, difunda y distribuya conocimiento cinematográfico. Tipologías de fuentes variadas y abundantes en las que el autor se ha apoyado y documentado ampliamente con dicho objetivo: bibliográficas, sonoras, audiovisuales, electrónicas, multimedia...; institucionales públicas y privadas; de medios de comunicación: cine, prensa, publicidad, radio, televisión, bibliotecas y servicios especializados universitarios (blogs, portales, canales, plataformas científicas...); internet; redes sociales tanto generales como especializadas...(López, 2014a, 2014c).

En suma, consideramos aquí como acepción más adecuada la conceptualización de Cinedocnet como red informativa, como red de conocimiento cinematográfico, de producción informativa sobre comunicación, información y documentación cinematográficas, como repositorio multimedia de acceso abierto, con dominio propio, cuya clasificación de contenidos se detalla en el epígrafe siguiente.

\section{Cinedocnet segunda etapa, 2014-...: estructura de contenidos}

La estructura del portal se desdobla fundamentalmente en dos grandes bloques informativos: noticias (actualidad, alertas, convocatorias...) y fuentes de información cinematográfica española (en una primera fase) e iberoamericana, en una etapa posterior. La base de datos de Cinedocnet remite a fuentes de información cinematográfica institucionales, bibliográficas, icónicas, sonoras, audiovisuales, multimedia, transmedia, electrónicas... de acuerdo con la siguiente clasificación de contenidos profesionales y temáticos: archivos, centros y servicios de documentación sonoros y audiovisuales, cinetecas, filmotecas, cinematecas, fonotecas, videotecas, mediatecas, bibliotecas, mediatecas, repositorios sonoros y audiovisuales; asociaciones, federaciones, fundaciones, institutos...; medios de comunicaciónn - cine, prensa, publicidad, radio, televisión, internet, redes sociales, bibliotecasarchivos-servicios especializados universitarios, agencias-gabinetes prensa-gabinetes comunicación...; congresos, jornadas, encuentros, mesas redondas...; derechos de autor, propiedad intelectual; distribución; exhibición; formación e investigación (instituciones, ámbitos, titulaciones, programas, proyectos, grado, posgrado...); 
patrimonio sonoro y audiovisual; producción: preproducción, producción, postproducción y equipo técnico-artístico (profesionales); promoción, festivales, premios, mercadotecnia; preservación, conservación y restauración; publicación y difusión informativa -fuentes generales de consulta o referencia (diccionarios, enciclopedias, bibliografías, directorios, repositorios, inventarios...), libros, revistas, estudios documentales y monográficos (crítica cinematográfica, guiones cinematográficos, historia, escenografía, iluminación, cámaras...), tesis doctorales, crítica cinematográfica, géneros cinematográficos, bases de datos, bancos sonido, grabaciones sonoras y audiovisuales, tesis doctorales, blogs, boletines electrónicos, canales, portales, plataformas, redes, listas, recolectores, servicios de alertas...-; y tecnologías (hardware, software, multimedia, transmedia...).

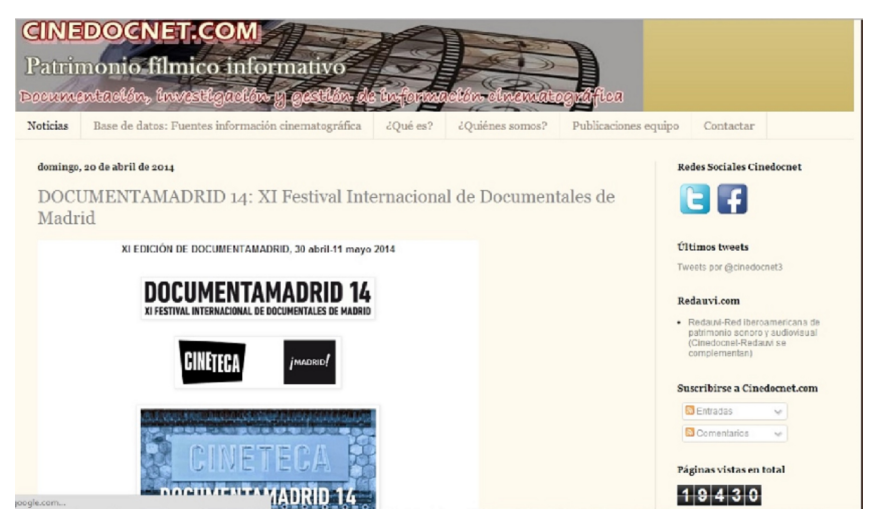

Figura 2. Cinedocnet-portada

Conviene destacar que esta clasificación de contenidos es sumamente amplia y engloba numerosos ámbitos de actuación relacionados con el patrimonio fílmico español. Por ejemplo, en el punto sobre Publicación-Difusión informativa, que reúne pertinentes instrumentos de producción, recuperación y divulgación cinematográficas, podrían incorporarse asimismo diversos estudios documentales y monográficos no enumerados - sólo se mencionan a título de ejemplo algunas temáticas-. Como por ejemplo un estudio monográfico sobre FormaciónInvestigación: metodología de trabajo del cineasta, del equipo técnico-artístico: ¿cómo se documenta, cómo investiga, cómo prepara su trabajo, qué fuentes de información utiliza, consulta archivos, visiona películas...? (Cinedocnet, 2012).

Y tampoco aparecen mencionados aquí otros documentos, pero sí contemplados en el portal, como directorios de instituciones, profesionales, revistas, tesis doctorales, recursos académicos muy diversos, filmotecas y archivos fílmicos, mediatecas, videotecas, etc. Debe quedar asimismo clarificado, una vez más, que el portal contemplará la presencia del cine y sus especializaciones en el marco de los medios de comunicación en su totalidad (de ahí, insistimos, el calificativo de "informativo"): prensa, publicidad, radio, televisión, redes sociales, internet... e incluso medios universitarios sonoros, audiovisuales y multimedia cinematográficos (López, 2014a).

Por otra parte, la estructura de contenidos y procedencia de los mismos que atañe a los dos grandes bloques informativos -Noticias y la base de datos Fuentes de información cinematográfica española-, incluye documentos procedentes del siguiente repertorio de tipología de fuentes informativas, especialmente aplicadas a la base de datos (Cinedocnet: base de datos, 2014).

En cuanto a fuentes institucionales, se recogen entre otras: Medios de comunicación y empresas informativas relacionadas; Red de documentación en medios de comunicación (Redocom, Ver cine) (2014); festivales cinematográficos; agencias de comunicación cinematográfica (pases y ruedas de prensa); filmotecas-cinetecas...; directorios-repertorios; promoción (festivales, premios...); universidades públicas y privadas (facultades de ciencias de la Información y comunicación, escuelas de comunicación...; formación virtual; bibliotecas universitarias, servicios especializados; archivos, bibliotecas, museos (información cinematográfica); fundaciones; asociaciones; congresos especializados, específicos sobre cine... Con respecto a fuentes bibliográficas y electrónicas, se recopilan entre otras: libros; obras de consulta o referencia (directorios, repertorios...); revistas especializadas; CDROM/DVD... Vídeos; redes sociales generales y específicas sobre información cinematográfica (por ejemplo "Cero en Conducta" (Educación y Cine) (2014); otras redes relacionadas, con referencias cinematográficas, como REBIUN (Red de bibliotecas universitarias) (2014), PARES (Portal de archivos españoles) (2014) o Red digital de colecciones de museos de España y Colecciones en red (Cer.es) (2014); Hispana (Europeana): Directorio de colecciones digitales españolas (2014); blogs, portales, sitios de interés... (El documentalista audiovisual, 2014; $\mathrm{Pa}$ peles de música, 2014; Portal de la comunicación, 2014; Plat.tv., 2014; Margenes.org, 2014; Dogma, 2014; UbuWeb, 2014); listas de distribución (Documentación y nuevas tecnologías en el cine español, 2014); Dialnet (2014)... 


\section{Divulgación, sistematización, normalización de contenidos: metadatos-programa indexator}

Cinedocnet es un proyecto de investigación y desarrollo social (integrado en un proyecto previo, Redauvi, como proyecto de red iberoamericana de patrimonio sonoro y audiovisual con la colaboración dee siete países iberoamericanos (Argentina, Bolivia, Colombia, Chile, España, México y Perú) con el objetivo de crear una red temática orientada a recuperar, recopilar y difundir el patrimonio cinematográfico de la región iberoamericana. Al igual que Redauvi se proponía, Cinedocnet realizará un diagnóstico próximamente, con la creación previa de un inventario que recupere las principales colecciones y fondos cinematográficos de los países participantes en el proyecto, con apertura a la posterior incorporación otros países de la región iberoamericana. Los medios de dichos países en cuyo ámbito se están aplicando los objetivos y contenidos del proyecto son fundamentalmente, en la etapa actual, ámbitos de comunicación y de empresa informativa en general, concretándose fundamentalmente en fonotecas, videotecas, fototecas, filmotecas-cinetecas-cinematecas y mediatecas.

Por destacar un ejemplo de institución de gran interés, sobre la que proporcionar información acerca de su proceso evolutivo y realizaciones en el ámbito mexicano de filmotecas, convendría aludir a la labor que viene desarrollando durante ya 50 años la Filmoteca de la UNAM, cuya directora general Guadalupe Ferrer intervino el 27 febrero de 2014 en el II TeleEncuentro internacional interactivo BICC Ronda14 (Ferrer, 2014).

En suma, la red informativa Cinedocnet, fundamentada en una plataforma informática de código abierto y software pertinente, apuesta por la construcción social del conocimiento basado en la interacción entre distintos grupos multidisciplinarios de profesionales y diferentes comunidades de usuarios y empresas que aportarán contenidos e investigación.

El objetivo fundamental del proyecto es recopilar, integrar, distribuir, intercambiar y difundir el estado de preservación del patrimonio sonoro y audiovisual en siete países de Iberoamérica para generar un conocimiento compartido y establecer un programa de formación para los archivistas, documentalistas y bibliotecarios de Iberoamérica. La red informativa Cinedocnet elaborará un informe en torno a la situación que presentan los archivos fílmicos en la región iberoamericana, convirtiéndose dicha red en un espacio para fomentar el conocimiento y empleo de métodos, técnicas, tecnologías, recomendaciones y estándares para la preservación del patrimonio cinematográfico iberoamericano. Se publicará un inventario de las principales colecciones o fondos documentales cinematográficos (patrimonio fílmico), quedando disponible para su consulta y descarga en la página web del proyecto.

En cuanto a beneficios resultantes, la Red informativa Cinedocnet supone asimismo una plataforma que ofrece documentación especializada, normas, estándares y recomendaciones internacionales para la salvaguarda del patrimonio fílmico de lberoamérica. Por otra parte, podría plantearse más adelante el establecimiento de un programa de formación y capacitación profesional a través de un programa de formación de formadores que tenga impacto en los países participantes, para lo que se contará con profesionales capacitados que puedan atender de forma integral los procesos documentales en un archivo cinematográfico.

Con respecto a catalogación y tratamiento documental de contenidos de Cinedocnet, para la descripción de las fuentes de información cinematográfica contempladas en la red, se utiliza un sistema de metadatos diseñado mediante el programa Indexator, basado en Dublin Core y RDF (Annemieke, 2003; Dublin Core, 2014; Gligorov, 2011; Metadata, 2014; Minglian, Xingxing, Jiuzhen, 2004). Indexator supone la recuperación, comunicación y difusión científica, pertinentes, de los recursos sonoros y audiovisuales cinematográficos alojados en el portal, con todavía mayores posibilidades de recuperación en función de sus canales de sindicación orientados hacia la comunidad de usuarios interesados. Sus potencialidades se refuerzan con su capacidad mailing masivo gracias a las excelencias del webcrawler Mbot, y sus repercusiones en la afiliación de las cuentas de twitter y Facebook (Blázquez, 2013a).

El propósito fundamental de Indexator es servir de instrumento canalizador de los objetivos de la futura red iberoamericana de patrimonio fílmico informativo, sustentada en la recuperación, recopilación, integración, catalogación y difusión de inventarios o repositorios de archivos sonoros y audiovisuales cinematográficos, y sus colecciones o fondos documentales accesibles en línea, en acceso abierto. Indexator ha sido elaborado como un sistema de indexación de contenidos multidisciplinares y polivalente, capaz de adaptarse a cualquier necesidad documental. Para mayor exhaustividad en las descripciones, el programa permite establecer esquemas de metadatos a voluntad del usuario, de forma tal que es posible aumentar el volumen descriptivo del análisis (López, 2014a). 


\section{Difusión informativa y sostenibilidad de cinedocnet}

Cinedocnet, a través del ámbito investigador en que está integrada (Servicio Documentación Multimedia) y con su propia denominación, está presente en las redes sociales generales Facebook (2014), Twitter (2014), LinkedIN, Google+ (2014) y especialmente YouTubeRedauvi (2014ab) y especializadas, tanto académico-científicas (Researchgate.net y Academia.edu, por ejemplo) como específicas sobre cine (Cero en Conducta, 2014ab).

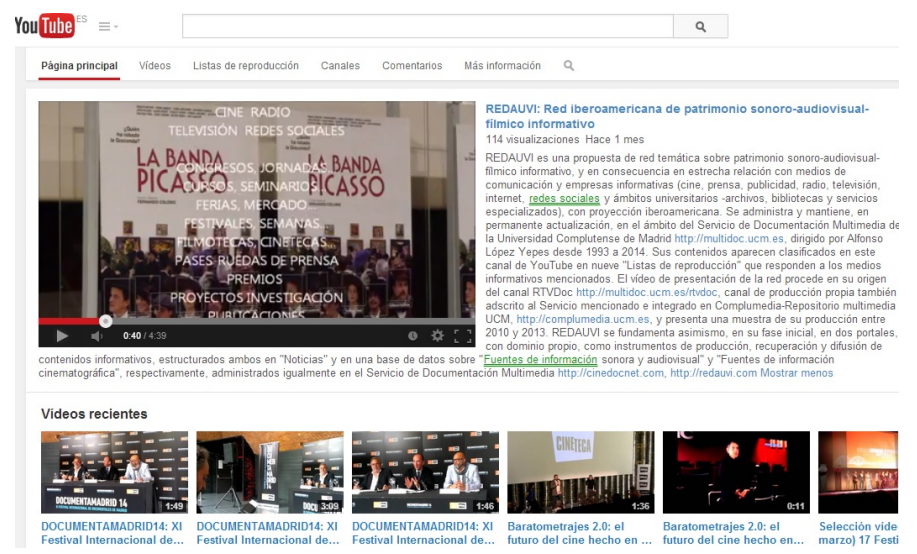

Figura 3. YouTube-Redauvi

La presencia de Cinedocnet en las redes sociales se caracteriza por la materialización de las siguientes acciones, que contribuyen eficazmente a su dinamización en la web social (López, 2014b):

1. No se trata de "estar por estar" en las redes (un error muy extendido), sino de tener definido claramente lo que se desea conseguir.

2. Público objetivo (usuarios reales) y análisis permanente de qué hacen otros sitios (instituciones, empresas) relacionados con el ámbito y conocimiento cinematográficos (usuarios potenciales).

3. Política editorial: cuidar los contenidos a publicar, que sean de interés para la comunidad académico-científico-investigadora, e incluso no sólo para el ámbito cinematográfico, sino también multidisciplinar.

4. El reto, en consecuencia: lograr la interacción con el público.

5. Cinedocnet está ampliamente representado en YouTube, en forma de canales ("listas de reproducción") clasificados por temáticas cinematográficas, las anteriormente relacionadas en el epígrafe 3. Conviene difundir dicha presencia a través de otras redes sociales.
6. Asimismo, anunciar (difundir) los eventos publicados en la sección "Noticias" de la red informativa (posts).

7. Crear encuestas de opinión en Facebook: por ejemplo, algo así como ¿qué les gustaría a los usuarios encontrar en la red? ¿están contentos? ¿qué echan de menos?.

8. Destacar curiosidades del fondo patrimonial en los diferentes perfiles.

9. Hacer recomendaciones sobre películas y documentales.

10.Aconsejar a los usuarios sobre métodos de conservación y gestión de su fondo personal.

11.Utilizar profusamente herramientas gratuitas para administrar diferentes rede sociales, como por ejemplo, una de las más conocidas y utilizadas: Hootsuite.

12.Actualización permanente de contenidos.

13. Reflexionar si se debe estar en todas las redes sociales, teniendo en cuenta que algunas de ellas no disponen de interacción alguna en el campo de interés.

14.Elaboración de cronogramas diversos sobre pasos a seguir en todo momento.

15. Medición de resultados. Existen muchas herramientas que permiten evaluar los resultados de las redes sociales, para saber si la estrategia que se ha definido está funcionado y se están obteniendo los objetivos previstos. Una de las preguntas fundamentales en este punto es: ¿Cómo podemos mejorar?. Algunas herramientas son, por ejemplo: Youtube analytics; Facebook: Likealyzer; Twitter: Socialbro.

16. En fin, es recomendable disponer de un plan de "crisis en línea", por si se llega a producir algún problema con los usuarios.

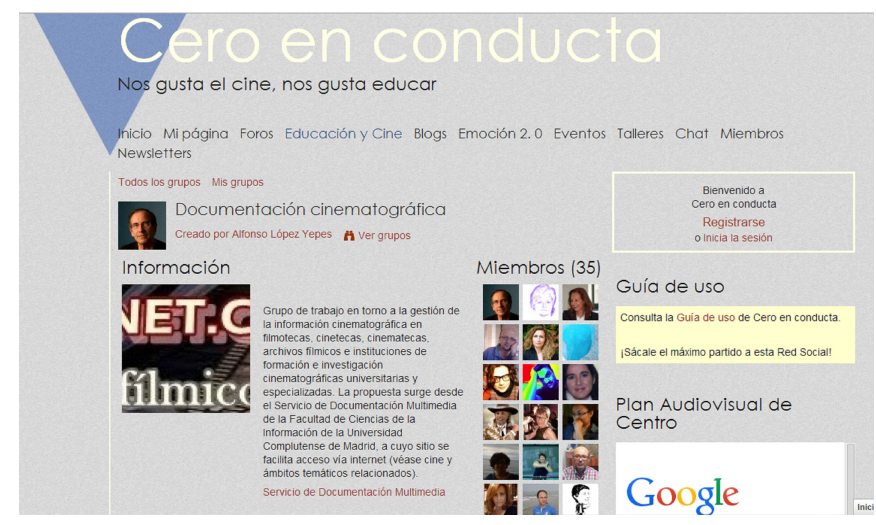

Figura 4. Red social Educación y Cine: Cero en Conducta 
Por otra parte, además de dicha presencia en redes sociales, el plan de difusión informativa de Cinedocnet se fundamenta, entre otras realizaciones, en la celebración de cursos y seminarios, participación en congresos nacionales e internacionales (el más reciente, $\mathrm{V}$ Congreso Internacional de Investigadores Audiovisuales (2014), con la consiguiente publicación de libros en colaboración, artículos, actas de congresos, eprints, etc. y otros ámbitos de difusión, como los especializados en formación virtual, como el Máster de documentación digital de la Universitat Pompeu Fabra (2014).

Otras manifestaciones de la presencia en el ámbito universitario de Cinedocnet y su canal RTVDoc se materializan, por ejemplo, en varias actuaciones como son los E-prints Complutense (E-prints Complutense, 2013) o la difusión informativa realizada también a través de RTVDoc del documental Perú: el reto de leer, proyecto de cooperación al desarrollo financiado por la UCM a través de su Biblioteca Central y realizado por la Plataforma de Divulgación Científica UCM (Plataforma Divulgación Científica, 2013). Actuación universitaria que se enmarca en el ámbito audiovisual y multimedia de los Servicios Audiovisuales de Universidades Españolas (SAVUES) a propósito de proyectos de coproducción de contenidos audiovisuales como $\mathrm{La}$ Universidad Responde. La difusión del conjunto de la coproducción se realiza a través de las webs de cada universidad, así como la de otros servicios audiovisuales o canales de televisión con el que se acuerde su difusión (SAVUES, 2013).

Con respecto a la sostenibilidad de la futura red temática de patrimonio fílmico informativo, se mantendrá una relación estrecha a nivel nacional e internacional con instituciones especializadas y muy estrechamente relacionadas, como por ejemplo la Federación Internacional de Archivos Fílmicos (FIAF) (2014), la Asociación Internacional de Archivos Sonoros y Audiovisuales (IASA) (2014), la Federación Internacional de Archivos de Televisión (FIAT) (2014), entre otras instituciones representativas también a nivel nacional en cada uno de los países e instituciones comprometidas en el desarrollo y evolución de la red temática.

Para mejorar las posibilidades de difusión informativa del portal Cinedocnet, se utiliza la herramienta webcrawler Mbot con su función de mailing masivo. En realidad, un webcrawler es un programa informático específicamente diseñado para rastrear la red de forma sectorial o completa, a partir de los enlaces y vínculos de las páginas web que analiza. En el caso de Mbot se sigue este patrón clásico para el análi- sis de enlaces y añade otras funciones derivadas, que facilitan las labores académicocientíficas y profesionales. Por ejemplo, generar un análisis de los formatos de archivos y páginas web encontrados, obtener información de las páginas más enlazadas, el análisis de los metadatos y meta-etiquetas encontrados, el análisis de co-enlaces, el establecimiento de rankings de recursos y páginas enlazados, el desarrollo de mapas de la web, entre otras múltiples aplicaciones (Blázquez 2012a).

Mbot fue creado en 2010 para cubrir las necesidades específicas de los investigadores en Documentación que se enfrentaban al estudio de la web desde el punto de vista cibermétrico. Este webcrawler constituye una parte importante de la cadena documental en la recuperación de información, dado que genera el corpus documental necesario sobre el que aplicar los distintos algoritmos de recuperación (Blázquez, 2014).

Mbot organiza y tabula la información automáticamente generando una base de conocimiento en la que se distribuyen y clasifican los tipos de páginas web según sus formatos y las referencias a los archivos de imagen, audio, vídeo. Por otra parte, almacena las meta-etiquetas estándar y los metadatos normalizados Dublin Core, para permitir estudios de recuperación de información y posicionamiento en buscadores.

Además, Mbot está diseñado para detectar canales de sindicación, redes semánticas y ontologías, de forma tal que puede realizar tareas de minería de datos especializadas, recabando información primaria a partir de un análisis en profundidad de la web. También es posible configurar Mbot, para la extracción masiva de correos electrónicos, códigos fuente, así como la indexación del texto completo de las páginas web que analiza. En 2013, el webcrawler Mbot se presenta en las XIII Jornadas Españolas de Documentación FESABID (Blázquez, 2013b), aportando un análisis de la web de la universidad española, generando también el primer mapa de la web universitaria española y sus realizaciones. Apostamos, en consecuencia, por su aplicación en el ámbito de Cinedocnet, en virtud de sus excelencias y sus posibilidades demostradas y experimentadas.

\section{Web semántica y patrimonio fílmico}

En la actualidad se están prodigando cada vez más los foros profesionales (congresos, jornadas, encuentros) en torno a web semántica y Linked Open Data, y su relación con instituciones patrimoniales (archivos, bibliotecas, museos, centros de documentación), los beneficios 
que les pueden aportar estas tecnologías y cómo se está trabajando con ellas y aplicándolas en el contexto de los datos abiertos.

Se hace referencia en este sentido, por ejemplo, a la Jornada que sobre Web semántica en archivos, bibliotecas y museos organizó en Madrid el 10 de marzo de 2014 la Fundación Ramón Areces (2014), en cuyo desarrollo se remitió a aplicaciones concretas sobre fondos documentales cinematográficos. En este sentido se mencionan algunos ámbitos de trabajo analizados por parte de ponentes en dicha Jornada, como por ejemplo la presentación de las bases de datos en línea de la Biblioteca Nacional Datos.bne.es 2.0 (2014), el portal de archivos españoles PARES y las colecciones museísticas (ya mencionados ambos con anterioridad) o, en fin, la labor que viene desarrollando en este campo de investigación la Fundación Ignacio Larramendi (2014). Precisamente sobre todo ello, con información bastante más ampliada, se hace eco Cinedocnet (2014).

\section{Conclusiones}

El proyecto surge en el ámbito universitario público español, como respuesta a un vacío y dispersión informativa en red sobre patrimonio cinematográfico. El concepto de patrimonio cinematográfico se contempla con motivo de este proyecto - además de como conservaciónpreservación-restauración (labor que fundamentalmente desarrollan las filmotecas) - especialmente desde el punto de vista de la comunicación, la información, la difusión cultural, incluso el conocimiento multidisciplinar. En consecuencia, se trata de una propuesta de red informativa de patrimonio fílmico, fundamentalmente en medios de comunicación y empresas informativas, de acceso abierto y proyección iberoamericana.

Tras un concienzudo estudio del estado de la cuestión, se concluye que en el ámbito iberoamericano (España, Latinoamérica, Brasil y Portugal) podrían existir accesibles en línea muchos más fondos cinematográficos y promoverse la interconexión de instituciones especializadas: filmotecas, cinetecas, cinematecas, archivos fílmicos y ámbitos estrechamente relacionados con la comunicación cinematográfica.

Además, la evolución de la web semántica y el Linked Open Data (datos entrelazados) repercutirá inevitablemente en el patrimonio fílmico: de hecho, ya existen desarrollos propios de esta tecnología.

Las acciones emprendidas (en constante desarrollo) sobre Cinedocnet aseguran una presen- cia relevante en redes sociales generalistas, académico-científicas y especializadas, con el objetivo final de convertirse en red social propia.

La difusión de los contenidos de Cinedocnet se efectúa mediante herramientas especializadas propias de la web social, amplia y convenientemente experimentadas.

La presencia de la Red informativa de patrimonio fílmico -y sus canales y herramientas propias de producción, recuperación y difusión informativas - también se hace patente en los ámbitos universitarios audiovisuales (bibliotecas y servicios especializados) y en actuaciones de los Servicios Audiovisuales de las Universidades Españolas (SAVUES) como La Universidad Responde y las plataformas de divulgación científica.

\section{Referencias}

Academia.edu. http://ucm.academia.edu/serviciodedocumenta cionmultimediamultidoc/Posts/395321/Consolidacion-deRedocom-2.0-Red-iberoamericana-de-documentacion-enmedios-de-comunicacion-http---mu (2014.04.25).

Amo García, Alfonso del (2006). La conservación cultural del patrimonio cinematográfico y la investigación científica. // Arbor: Ciencia, pensamiento y cultura. 182:717, 9-16.

Amo García, Alfonso del (2012). El centro de conservación y restauración de la Filmoteca Española. // Archivos de la Filmoteca. 72.

Annemieke de Jong (2003). Metadata in the audiovisual production environment: An Introduction (e-book). https://www.prestocentre.org/library/resources/metadataaudiovisual-production-environment (2014.04.11).

Asociación Internacional de Archivos Sonoros y Audiovisuales (IASA).http://www.iasa-web.org/ (2014.04.25).

Blázquez Ochando, Manuel (2012a). Análisis webmétrico de los medios de comunicación brasileños: prensa, radio y televisión. // I Seminario Hispano Brasileño de Biblioteconomía y Documentación (Madrid, 28-30 noviembre). http://eprints.rclis.org/19033/ (2014.04.11)

Blázquez Ochando, Manuel (2014). Nuevos retos de la tecnología web crawler para la recuperación de información. // MEI. II:4:7, 115-128. http://www.metodosdein formacion.es/mei/index.php/mei/article/viewFile/IIMEI4N7-115128/788 (2014.04.11).

Blázquez Ochando, Manuel. (2013a). Técnicas avanzadas de recuperación de información: procesos, técnicas y métodospp.1-117. ISBN 978-84-695-8030-1. (Libros y manuales de la Documentación; 4). http://mblazquez.es /wp-content/uploads/ebook-mbo-tecnicas-avanzadas-re cuperacion-informacion1.pdf (2014.04.11). V. también http://mblazquez.es/category/proyectos/aplicaciones-pro fesionales (2014.04.11).

Blázquez Ochando, Manuel. (2013b). Desarrollo tecnológico y documental del webcrawler Mbot: prueba de análisis web de la universidad española. // XIII Jornadas Españolas de Documentación, Fesabid (Toledo, 21-24 mayo). 125-136. http://www.fesabid.org/toledo2013/actasde-las-jornadas (2014.04.11).

Brausin, Dora; López Yepes, Alfonso; Rodriguez Reséndiz, Perla (2012). Red de archivos sonoros, audiovisuales y multimedia con proyección iberoamericana (ColombiaMéxico-España). // II Conferencia Internacional sobre Bibliotecas y Repositorios Digitales - BIREDIAL'12, 13- 
16 noviembre 2012. Barranquilla (Colombia), Universidad del Norte, 2012. http://eventos.uninorte.edu.co/index .php/biredial/biredial2012/paper/view/386 (2014.04.11).

Camarero Gómez, M. Gloria (2012). Patrimonio Cinematográfico en España: conservación, restauración y difusión. // I Congreso Internacional "El patrimonio cultural y natural como motor de desarrollo: investigación e innovación": [DVD] /. A. Peinado Herreros, coord. 2012. 645657

Cero en Conducta "Educación y Cine". http://ceroenconduc ta.ning.com/ (2014.04.25)

Cero en Conducta: "Documentación cinematográfica". http://ceroenconducta.ning.com/group/documentacion-ci nematografica (2014.04.25).

Cero en Conducta: "Educación y Cine". http://ceroenconduc ta.ning.com/ (2014.04.25).

Cinedocnet (2012). http://cinedocnet.com. Formación-Investigación: metodología de trabajo del cineasta, del equipo técnico-artístico: ¿cómo se documenta, cómo investiga, cómo prepara su trabajo, qué fuentes de información utiliza, consulta archivos, visiona películas...? http://www. cinedocnet.com/search/label/metodolog\%C3\%ADa\%20 cineasta (2014.04.11).

Cinedocnet (2014). Web semántica en archivos, bibliotecas y museos: referencias a patrimonio cinematográfico español (25.04.2014). http://www.cinedocnet.com/2014/04/web-se mantica-en-archivos-bibliotecas-y.html (2014.04.25).

Cinedocnet: base de datos "Fuentes de información cinematográfica. http://www.cinedocnet.com/p/filmotecas-cinete cas.html http://mblazquez.es/redauvi/ (2014.04.25).

Cinedocnet: Patrimonio fílmico informativo. http://cinedocnet .com (2014.04.25)

Datos.bne.es 2.0 (2014). http://www.slideshare.net/Daniel VilaSuero/datosbnees-20 (2014.04.25).

DIALNET: Servicio de alertas sobre publicación de contenidos científicos.http://dialnet.unirioja.es/ (2014.04.25).

Docma. http://docma.es/ (2014.04.25).

Documentación y nuevas tecnologías en el cine español (CINEDOC).http://www.rediris.es/list/info/cinedoc.html (2014.04.25).

Dublin Core (2014). Metadata Iniciative. http://dublincore.org /documents/dcmi-terms/, http://dcpapers.dublincore.org/ pubs/article/viewFile/776/772 (Consulta 2014.04.11).

E-prints Complutense (2013). López Yepes, Alfonso. Vídeo Los ilusos, de Jonás Trueba: pase y rueda en prensa en Cineteca Matadero Madrid, 10 abril 2013; Vídeos de producción propia sobre Documentación, formación, investigación cinematográficas, 2007-2013; Vídeos de producción propia sobre Metodología de la investigación científica/cómo se hace una tesis doctoral. (RTVDocUCM). http://eprints.ucm.es/view/types/video.html. Consulta: 2014.04.11).

El documentalista audiovisual. http://www.eldocumentalistau diovisual.com (2014.04.25)

Facebook. https://www.facebook.com/cinedocnet.cine?fref= ts (2014.04.25)

Federación Internacional de Archivos de Televisión (FIAT). http://fiatifta.org/ (2014.04.25)

Federación Internacional de Archivos Fílmicos (FIAF). http://www.fiafnet.org/es/ (2014.04.25).

Ferrer, Guadalupe (2014). La Filmoteca de la UNAM. // La preservación y difusión de los patrimonios sonoros y audiovisuales en Iberoamérica. II TeleEncuentro internacional interactivo BICC Ronda14. http://www. filmoteca.unam. mx/amiba/, http://teibicc2014.blogspot.com.es/p/2-tele-en cuentro-audiovisual.html (2014.04.11).

Franco Lorenzana, Julián (2012). Rescatando sombras. Madrid, Asociación de Amigos de la Filmoteca Españo-
la-AAFE, 2012. http://rescatando sombras.es, http://www .cinedocnet.com/2012/05/filmoteca-conservacionrestau racion.html (15.04.2014).

Fundación Ignacio Larramendi (2014). http://www.larramendi .es/i18n/estaticos/contenido.cmd?pagina=estaticos/inicio (2014.04.25).

Fundación Ramón Areces (2014). Jornada que sobre Web semántica en archivos, bibliotecas y museos. http://www.fundacionareces.es/fundacionareces/cargarA plicacionAgendaEventos.do?verPrograma=1\&identifica dor=1634 (2014.04.25).

García Casado, Pablo; Alberich Pascual, Jordi (2014). Filmotecas en la encrucijada. Función y expansión de la actividad filmotecaria en el nuevo escenario digital. // El Profesional de la Información. 23:21. http://www .elprofesionaldelainformacion.com/contenidos/2014/ener o/07.html (2014.04.11).

Gligorov, Riste et al. (2011). On the role of user-generated metadata in audio visual collections. // K-CAP '11. Proceedings of the sixth international conference on Knowledge capture. 145-152. New York, NY, USA: ACM http://dl.acm.org/citation.cfm?id=1999702 (2014.04.11).

Google+. https://plus.google.com/102271785885923949996/ posts (2014.04.25).

Hispana (Europeana). Referencias cinematográficas en: Arquivo da Memoria Social de Vilagarc ia de Arousa, Ayuntamiento de Vilagarcía de Arousa; Biblioteca Virtual Miguel de Cervantes, Fundación Biblioteca Virtual Miguel de Cervantes Saavedra; E-prints Complutense, Biblioteca Universidad Complutense; Filmoteca Canaria Gobierno de Canarias; Filmoteca Digital, Filmoteca Española; Fondos digitalizados del Archivo Municipal de Castellón, Archivo Municipal de Castellón.http://hispana .mcu.es/es/estaticos/contenido.cmd?pagina=estaticos/pr esentacion (2014.04.25).

López Yepes, Alfonso (2008). Filmotecas y archivos filmicos en línea: producción, difusión, interconexión y posicionamiento en Internet. // Scire: Representación y organización del conocimiento. 14:2, 41-64. http://www.ibersid. eu/ojs/index.php/scire/article/view/1750. (2014.04.11).

López Yepes, Alfonso (2013a). El cine en español a propósito de REDAUVI: Red iberoamericana para la integración, preservación y difusión del patrimonio sonoro y audiovisual. // II Congreso Internacional Historia, literatura y arte en el cine en español y portugués: De los orígenes a la revolución tecnológica del siglo XXI, Salamanca, 26-28 de junio de 2013 / coord. por Emma Camarero Calandria, María Marcos Ramos, 2013. 552-558. $\mathrm{http}: / /$ dialnet.unirioja.es/servlet/articulo?codigo $=4332497$ (2014.04.11)

López Yepes, Alfonso (2013b). Vídeos de producción propia sobre Documentación, formación, investigación cinematográficas, 2007-2013. // Canal RTVDoc-UCM). http://eprints.ucm.es/23773/ (2014.04.11).

López Yepes, Alfonso (2014a). Portal de investigación y divulgación de fuentes de información cinematográfica española (Cinedocnet): hacia una red iberoamericana de patrimonio fílmico informativo. // Francisco Ubierna Gómez y Javier Sierra Sánchez (Coordinadores). Miscelánea sobre el entorno audiovisual en 2014 (V Congreso Internacional de Investigadores Audiovisuales, 24-25 abril 2014). Madrid, Editorial Fragua. 953-971.

López Yepes, Alfonso (2014b). Entrevista a Alfonso López Yepes sobre Cinedocnet. // Cristòfol Rovira; Mari Carmen Marcos; Lluís Codina (dir.). Máster en Buscadores. Barcelona: Área de Ciencias de la Documentación. Departamento de Comunicación. Universidad Pompeu Fabra. http://www.documentaciondigital.org (18.04.2014).

López Yepes, Alfonso (2014c). Audiovisual en línea en la universidad española: bibliotecas y servicios especiali- 
zados (una panorámica) (2014). // Icono14. (En prensa). http://www.icono14.net/ojs/index.php/icono14 (2014.04.11)

López Yepes, Alfonso; Arratia, Amira; Brausin, Dora; De Giusti, Marisa; Martínez, Silvia; Rodríguez, Perla Olivia; Suárez, Patricia (2014). Patrimonio sonoro y audiovisual: propuesta de creación de una red temática iberoamericana. // Innovación didáctica al servicio de los profesionales en ciencias de la información (Congreso Virtual vía Internet), septiembre 2013. Madrid-España, La Plata-Argentina.

Margenes.org. http://www.margenes.org/ (2014.04.25).

Máster de documentación digital, Universitat Pompeu Fabra. http://www.documentaciondigital.org (2014.04.25).

Metadata for Audiovisual Materials and its Role in Digital Projects (2014). VRACORE: a data standard for the description of images and works of art and culture. http://www.vraweb.org/projects/vracore4/ (12.04.2014).

Minglian, Duan; Xingxing, Yao; Jiuzhen, Zhang (2004). DC2004. A study on audiovisual metadata. http://dcpapers.dublincore.org/pubs/article/viewFile/776/ 772 (13.04.2014).

Monasterio, José Enrique (2005). Investigación, conservación, restauración, documentación y difusión aplicadas al cine. Recomendaciones internacionales y legislación nacional y autonómica. Cuadernos de Documentación Multimedia. 16. http://multidoc.rediris.es/cdm/viewarticle .php?id=40 (13.04.2014)

Oliván Plazaola, Montserrat (2000). Patrimonio cultural, películas cinematográficas y Depósito Legal. // Boletín de la Asociación Andaluza de Bibliotecarios. 15:60 . 4556.

Papeles de Música. http://papelesdemusica.wordpress.com/ (2014.04.25).

Plat.tv. http://plat.tv/ (2014.04.25).

Plataforma Divulgación Científica (2013). Documental Perú: el reto de leer. Madrid: Biblioteca UCM, Cooperación Bi- bliotecaria. http://www.plataformadivulgacionucm.com/ $? p=2517$ (2014.04.12).

Portal de archivos españoles (PARES).http://pares.mcu.es/ (2014.04.25).

Portal de la Comunicación. http://www.portalcomunicacion. $\mathrm{com} /(2014.04 .25)$

Red de bibliotecas universitarias (REBIUN). http://www.re biun.org/Paginas/Inicio.aspx (2014.04.25).

Red de documentación en medios de comunicación. http://multidoc.rediris.es/redocom (2014.04.25).

Red digital de colecciones de museos de España y Colecciones en red (Cer.es). http://ceres.mcu.es/pages/ SimpleSearch?index=true (2014.04.25).

Red iberoamericana de patrimonio sonoro y audiovisual.http://redauvi.com (2014.04.25).

Servicio de Documentación Multimedia. Departamento de Biblioteconomía y Documentación, Universidad Complutense de Madrid. http://multidoc.ucm.es (2014.04.25).

Twitter. https://twitter.com/cinedocnet3 (2014.04.25).

UbuWeb. http://www.ubu.com/ (2014.04.25).

$\checkmark$ Congreso Internacional de Investigadores Audiovisuales Celebrado en la Escuela Universitaria de Diseño, Innovación y Tecnología (ESNA en Madrid el 24-25 abril 2014, bajo la organización de dicha escuela y la Asociación Científica Icono 14:I. http://www.cinedocnet.com/ 2014/04/v-congreso-internacional-de.html (2014.04.25).

YouTube-Redauvi . https://www.youtube.com/user/alyepes1/ playlist (2014.04.25).

Youtube-Redauvi. https://www.youtube.com/user/alyepes1 (2014.04.25).

Enviado: 2014-04-26. Segunda versión: 2014-06-23. Aceptado: 2014-06-23. 